Fizika Nizkikh Temperatur, 1998, v. 24, No 9, p. 856-860

\title{
Formation and growth dynamics of domains under phase transitions in an external field
}

\author{
L. I. Stefanovich \\ Physics and Engineering Institute, National Academy of Sciences of Ukraine, \\ 72 R. Luxemburg St., 340114, Donetsk, Ukraine \\ E-mail: listef@host.dipt.donetsk.ua \\ Received March 16, 1998
}

\begin{abstract}
The formation and the growth dynamics of $180^{\circ}$-domains in ferroelectrics in external field are investigated with the use of the statistical approach within the Ginzburg-Landau model. It is shown that despite the polarizing role of an external field the formation of an intermediate polydomain structure is found to be more preferable than immediate transfer to the monodomain ordering state.
\end{abstract}

PACS: 64.60.-i, 64.80.-v

\section{Introduction}

The fact that the really observed structure of solids is often nonequilibrium is largely determined by the dynamics of transformations occurring there. These transformations may have the character of both phase separation [1] and ordering [2]. In multicomponent alloys both of the aforesaid processes can proceed simultaneously [3]. In this paper we are concerned with the ordering processes and in large measure their dynamic aspect, which consists in tracing the paths of establishing one or another stable or metastable state and in revealing the reasons influencing this choice.

The structural phase transitions in ferroelectrics associated with the appearance of macroscopic regions of the crystal where the spontaneous electric polarization is not equal to zero are the basic object of our investigation in this work.

To characterize quantitatively the state change of a system passing through the critical temperature point $T_{c}$ of a phase transition, one or more values called order parameters are introduced. In the case of the ferroelectric phase transition the projection of the polarization vector on a certain crystallographic direction is used as a long-range order parameter.

It is known [4] that, in the absence of external field at the temperatures below $T_{c}$, i.e., in lowsymmetrical phase, the states correspond to the different (with respect to the sign) values of the order parameter $\pm \eta$. In the early stages of the ordering, when the relaxation of the short-range order has already been proceeded basically, the appearance of the structures of the type $+\eta$ or $-\eta$ proves to be quite accidental and therefore the regions of both the signs $\pm \eta$ (called usually $180^{\circ}$ domains) must exist in different points of the crystal. It is obvious that the spatial size of the domains is assumed to be much larger than the lattice parameter. As was shown by us earlier [2], the evolution of these inhomogeneities of the order parameter in the absence of the external field will proceed along one of two basic paths, depending on the initial conditions - either the formation of a single-domain state or the formation of a polydomain structure followed by increasing (for diffusion time) the spatial scale of this structure up to the crystallite size.

Our goal is to clear up how the long-range order evolution character will change in an initially disordered system if at the moment of quenching is finished some external field $\varepsilon$ conjugating with the order parameter $\eta$ will be imposed on it rapidly enough. The question is, if the homogeneous (monodomain) ordering will occur, or can a sufficiently developed polydomain structure formed, in some situations? In the case of a ferroelectric an uniform steady-state electric field should be meant when speaking about an external field. The time of its establishing $\left(\tau_{\varepsilon}=\varepsilon / \dot{\varepsilon}\right)$ is considered to be small in comparison with the time of the forming $\left(\tau_{d}\right)$ and certainly it is much less than the time of coalescence $\left(\tau_{c}\right)$ of the domain structure $\left(\tau_{\varepsilon}<<\tau_{d}<<\tau_{c}\right)$. 


\section{General description of the model}

In order to describe the ordering within the framework of Landau theory we shall assume further that, despite the proximity of temperature to the critical one $\left(T_{c}-T\right) / T_{c}<1$ the system lies outside the fluctuation region. In this situation a nonequilibrium addition to the thermodynamic potential in the presence of external field can be presented in the form of Ginzburg-Landau functional [5]:

$$
\Phi\{\eta, \nabla \eta\}=\int\left[\frac{1}{2} A \eta^{2}+\frac{1}{4} B \eta^{4}+\frac{1}{2} \delta(\nabla \eta)^{2}-\eta E\right] d V
$$

Here the coefficient $A$ is proportional to $\left(T_{c}-T\right)$. Since the characteristic energy scale in this problem is $T_{c}$ it may be considered that $B \sim T_{c}$, and $\delta \sim T_{c} r_{0}^{2}$, where $r_{0}$ is the interaction radius; i.e. the value of the order of interatomic distance; $E$ is the external field, which we believe for the simplicity to be homogeneous one.

In order to describe the dynamics of nonequilibrium system we shall use the Landau-Khalatnikov equation [2] for a nonconserved order parameter:

$$
\frac{\partial \eta}{\partial t}=-\gamma \frac{\delta \Phi}{\delta \eta}
$$

where $\gamma$ is the kinetic coefficient which can be represented in the form $\left(\tau T_{c}\right)^{-1}$, so $\tau$ can be interpreted to be the time required for an elementary rearrangement of the system (for example, a displacement of an atom or interchange of neighboring atoms).

If, now, distance is measured in units of $r_{0}$ and time in units of $\tau$, then Eq. (2) can be written, in view of Eq. (1), in the form:

$$
\partial \eta / \partial t=\Delta \eta+\alpha \eta-\eta^{3}+\varepsilon
$$

Here $\Delta$ is the Laplacian and we have introduced two parameters: $\alpha=\left(T_{c}-T\right) / T_{c}$ is a dimensionless parameter that characterizes the proximity of temperature $T$, up to that of the specimen cooled, to the temperature of ordering $T_{c} ; \varepsilon=E / T_{c}$ is the external field in corresponding units.

It should be emphasized that the initial state of order-disorder system must be given statistically, considering that, first, inhomogeneities of an order parameter are formed as a result of rapid cooling of specimen randomly arranged in space; second, there are thermal fluctuations of order parameters all along. The spatial scale of appropriate inhomogeneities is assumed to be much larger than the lattice parameter.
To solve the problem it is necessary to have an initial condition to Eq. (3), i.e. the meaning of order parameter $\eta(\mathbf{r}, t)$ at the initial moment of time should be given, $\eta(\mathbf{r}, 0) \equiv \eta_{0}(\mathbf{r})$. Since this initial function is random, the order parameter is a random function of coordinates for $t \neq 0$ as well. Therefore, Eq. (3) will be describe spatial-time evolution of the order parameter random field operating in the spatially uniform (and determinate) field $\varepsilon$.

\section{The derivation of basic equations}

To describe the relaxation processes taking place in the system undergoing the phase transition, there is no need to know the spatial distribution of the order parameter $\eta(\mathbf{r}, t)$ in detail over the total specimen. Therefore, we shall deal with the search for the main physically significant characteristics of this function in the subsequent discussion, such, for example, as the average (over crystalline grain) value of the order parameter $\langle\eta(\mathbf{r}, t)\rangle \equiv \bar{\eta}(t)$ and the two-point correlation function

$$
\left\langle\xi(\mathbf{r}, t) \xi\left(\mathbf{r}^{\prime}, t\right)\right\rangle \equiv K(\mathbf{s}, t), \quad \mathbf{s}=\mathbf{r}-\mathbf{r}^{\prime},
$$

where we have introduced the centered order parameter $\xi(\mathbf{r}, t) \equiv \eta(\mathbf{r}, t)-\bar{\eta}(t)$, and have used the standard assumption that the order parameter field is statistically uniform.

The equations for $\bar{\eta}(t)$ and $K(\mathbf{s}, t)$ are derived from the basic equation (3) both by averaging the equation itself and by averaging after premultiplying both sides of Eq. (3) by $\eta\left(\mathbf{r}^{\prime}, t\right)$.

To obtain a closed system of equations for $\bar{\eta}(t)$ and $K(\mathbf{s}, t)$, the possible asymmetry are neglected here, i.e., we suppose that

$$
\left\langle\xi^{2}(\mathbf{r}) \xi\left(\mathbf{r}^{\prime}\right)\right\rangle=0
$$

for all the $\mathbf{r}$ and $\mathbf{r}^{\prime}$. For correlation function of the type $\left\langle\xi^{3}(\mathbf{r}, t) \xi\left(\mathbf{r}^{\prime}, t\right)\right\rangle$ we shall use the unlinking of the form

$$
\begin{gathered}
\left\langle\xi^{3}(\mathbf{r}, t) \xi\left(\mathbf{r}^{\prime}, t\right)\right\rangle=\left\langle\xi^{2}(t)\right\rangle\left\langle\xi(\mathbf{r}, t) \xi\left(\mathbf{r}^{\prime}, t\right)\right\rangle \equiv \\
\equiv K(0, t) K(\mathbf{s}, t) .
\end{gathered}
$$

One of the justifications for this procedure is the availability of only one spatial scale in the problem considered.

It would appear natural, then, that the functional dependence of the fourth order correlation function $\left\langle\xi^{3}(\mathbf{r}) \xi\left(\mathbf{r}^{\prime}\right)\right\rangle$ of $\left|\mathbf{r}-\mathbf{r}^{\prime}\right|$ would be an accurate copy the functional dependence of the distance between points $\mathbf{r}$ and $\mathbf{r}^{\prime}$ for the second order correlation function $\left\langle\xi(\mathbf{r}) \xi\left(\mathbf{r}^{\prime}\right)\right\rangle \equiv K\left(\left|\mathbf{r}-\mathbf{r}^{\prime}\right|\right)$. It has been known that for the Gaussian random field the 
unlinking (6) will be an accurate one, if the righthand side of Eq. (6) is multiplied by a coefficient which is equal to three. In our case the Eq. (6), without any doubt, is an approximation, in which the choice of the coefficient is determined by the fact that in the problem considered, for long times in particular, the one-point distribution function is significantly different from Gaussian form. It follows from physical considerations based on the equivalency of states that are equal in magnitude but opposite in sign, of order parameters, that for the sufficiently long times $\left(t>>\alpha^{-1}\right)$ are close to the curve with two sharp maxima at the equilibrium values of the order parameter. Here, of course, we are dealing with a centered order parameter $\xi$. As it is immediately evident from the calculation, for such a distribution function the coefficient mentioned above is close to unity.

As a consequence of Eq. (3) and assumptions (5) and (6), we obtain, finally, the system of equations for the functions: $\bar{\eta}(t)$ and $K(\mathbf{s}, t)$

$$
\left\{\begin{array}{l}
\frac{d \eta}{d t}=\frac{1}{2}\left(\alpha \bar{\eta}-3 K(0, t) \bar{\eta}-\bar{\eta}^{3}+\varepsilon\right), \\
\frac{\partial K(\mathbf{s}, t)}{\partial t}=\Delta K(\mathbf{s}, t)+\left[\alpha-K(0, t)-3 \bar{\eta}^{2}\right] K(\mathbf{s}, t) .
\end{array}\right.
$$

The system of Eqs. (7), (8) contains two physically meaningful parameters: $\alpha$ and $\varepsilon$. Owing to the nonlinearity contained in the right-hand sides of Eqs. (7), (8), our system cannot be solved by analytical methods. However, as shown in [2], due to the distinctive degeneracy, the similar system can be reduced to the system of nonlinear ordinary differential equations for the average value of order parameter $\bar{\eta}(t)$ and dispersion of it $D=D(t)$ with the help of the Fourier transformation of the Eq. (8) on the spatial variable $\mathbf{s}$

$$
\left\{\begin{array}{l}
\frac{d \eta}{d t}=\frac{1}{2}\left[\left(\alpha-3 D-\bar{\eta}^{2}\right) \bar{\eta}+\varepsilon\right], \\
\frac{d D}{d t}=\left(\alpha_{\mathrm{eff}}(t)-D-3 \bar{\eta}^{2}\right) D,
\end{array}\right.
$$

where the following notations are introduced: $D=D(t)=K(0, t)$;

$$
\alpha_{\mathrm{eff}}(t) \equiv \alpha-1 / r_{c}^{2}(t) .
$$

Here we have used a natural determination of the correlation radius $r_{c}(t)$ by Fourier transform, $\tilde{K}(\mathbf{q}, t)$, of the correlation function:

$$
\frac{1}{r_{c}^{2}(t)} \equiv \int q^{2} \tilde{K}(\mathbf{q}, t) d^{3} q / \int \tilde{K}(\mathbf{q}, t) d^{3} q
$$

As it is shown in [2], the correlation radius at an arbitrary moment of time is determined by the value of the correlation function $K(\mathbf{s}, t)$ at the initial moment of time, i.e. $K(\mathbf{s}, 0)$. The last must be specified as the initial condition of the problem. The temporal dependence of the coefficient $\alpha_{\text {eff }}$, in accordance with $(10)$, is determined completely by the evolutionary character of the system correlation radius $r_{c}(t)$ (in our case it is associated with the characteristic spatial scale of ordered region or domain size).

For all the «acceptable» initial correlation functions, $K(\mathbf{s}, 0)$, as it is shown in [2], the temporal dependence of the correlation radius $r_{c}(t)$ has the form:

$$
r_{c}(t)=\sqrt{r_{c}^{2}(0)+2 t / 3},
$$

where $r_{c}(0)$ is the correlation radius of the system at the initial moment of time $(t=0)$. The last formula confirms the well-known conclusion that domain sizes grow with time according to a diffusion law in proportion to $\sqrt{t}$ (provided, of course, that $\left.t>>r_{c}^{2}(0)\right)$. Thus, the system of equations (9) for $\bar{\eta}(t)$ and $D(t)$, in the terms of the Eqs. (10), (12), takes the form

$$
\left\{\begin{array}{l}
\frac{d \bar{\eta}}{d t}=\frac{1}{2}\left[\left(\alpha-3 D(t)-\bar{\eta}^{2}\right) \bar{\eta}+\varepsilon\right], \\
\frac{d D(t)}{d t}=\left[\alpha-\left(2 / 3 t+r_{c}^{2}(0)\right)^{-1}-D(t)-3 \bar{\eta}^{2}\right] D(t) .
\end{array}\right.
$$

\section{Asymptotic behavior of system for long-times}

It is of greatest interest to study the system of Eqs. (13) close to the ordering temperature $T_{c}$, when $\alpha<<1$ and the initial correlation length is not too large, so that the condition $r_{c}^{2}(0)<<(1 / \alpha)<d^{2}$ can satisfied, where $d$ is the characteristic crystallite size (we recall that time is measured in units of $\tau$ and distance is measured in units of $r_{0}$, i.e., the interatomic interaction length). Then, asymptotically, i.e. at times $t>>1 / \alpha$, the system of Eqs. (13) transforms into a system of equations with constant coefficients

$$
\left\{\begin{array}{l}
\frac{d \bar{\eta}}{d t}=\frac{1}{2}\left[\left(\alpha-3 D(t)-\bar{\eta}^{2}\right) \bar{\eta}+\varepsilon\right], \\
\frac{d D(t)}{d t}=\left(\alpha-D(t)-3 \bar{\eta}^{2}\right) D(t),
\end{array}\right.
$$

with the initial conditions

$$
\bar{\eta}(0)=\bar{\eta}_{0} ; \quad D(0)=D_{0} .
$$


The solution of the system of Eqs. (14) with initial conditions (15) allows us to obtain information about the closing stages of the ordering process.

Let us perform qualitative analysis of the system (14) with use of the concept of phase pattern [6] (in the present case, in the variables $\bar{\eta}$ and $D$ (see Fig. 1).

As it is shown, the singular (stationary) points of the system (14) can be found from the fact that $(d \eta / d t) \rightarrow 0$ and $(d D / d t) \rightarrow 0$ as $t \rightarrow \infty$; therefore,

$$
\left\{\begin{array}{l}
\varepsilon+\alpha \bar{\eta}-3 D \bar{\eta}-\bar{\eta}^{3}=0 \\
\left(\alpha-D-3 \bar{\eta}^{2}\right) D=0
\end{array}\right.
$$

This is a system of two algebraic equations in variables $\bar{\eta}$ and $D$. The roots of the above-mentioned system yield the coordinates of singular points in the plane $(\bar{\eta}, D)$.

For $\alpha<0$, i.e. at a temperature above the critical point, there is only one singular point, which is a stable node. The coordinates of this point for the small $\varepsilon$ are $\bar{\eta}=\varepsilon / \alpha \mid, D=0$. This means that irrespectively of the initial conditions, the system will pass into a disordered state. First of all, it should be noted here that, even though the field $\varepsilon$ is as small as possible, the average value of order parameter $\bar{\eta}$ becomes nonvanishing everywhere over the temperature region. Owing to the external field, among other things, the phase transition appears to be «blurred», i.e., it takes place some temperature interval away from critical point $T_{c}$.

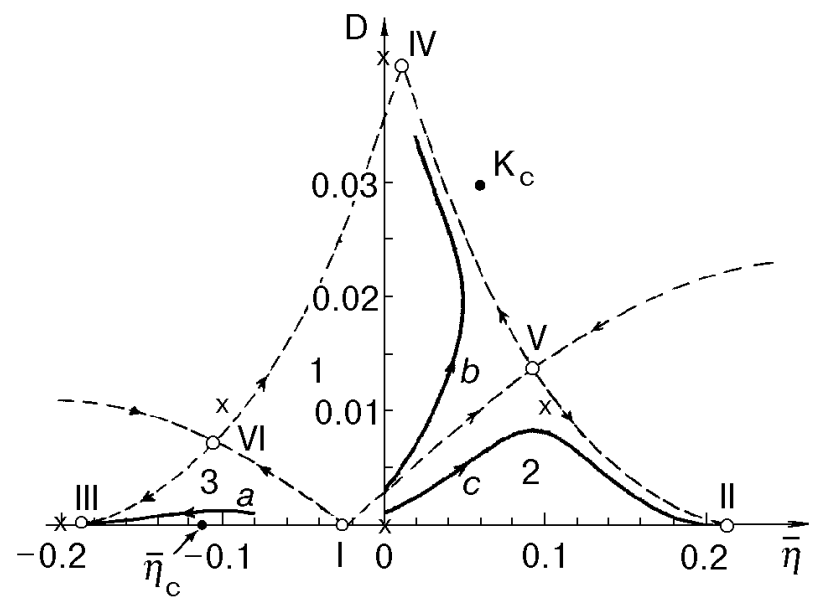

Fig. 1. Phase pattern of order-disorder system. The singular points of the system (14) at $\varepsilon=0$ are marked by symbol $(X)$; the singular points (I-VI) for the system (14) at $\varepsilon \neq 0$ are marked by circles ( $\bigcirc)$ and separatrices are indicated by dotted lines. The bifurcation points (at $\varepsilon=\varepsilon_{c}$ ) for the system (14) $\left(\eta_{c}\right.$ and $\left.K_{c}\right)$ are shown by black circles $(\bullet)$. Here solid lines $a-c$ are the phase trajectories as a result of numerical integrating of the total system (13) for $\alpha=0.04 ; \varepsilon=0.001 ; r_{c}(0)=10$ at different initial conditions $\left\{\bar{\eta}_{0}, D_{0}\right\}:\{-0.08 ; 0.001\}(a) ;\{0 ;$ $0.003\}(b) ;\{0 ; 0.001\}(c)$
For $\alpha>0$, i.e. at $T<T_{c}$, and not-too-strong field there is a whole system of singular points. However, only the points located in the upper half-plane of the phase pattern (in variables $\bar{\eta}, D$ ) will have physical meaning. Let us analyze, initially, the positions of the singular points in the case of weak field $\left(\varepsilon<<\alpha^{3 / 2}\right)$.

The first singular point I $[\bar{\eta}=-(\varepsilon / \alpha), D=0]$, which corresponds to a homogeneous disordered state, is an unstable node (Fig. 1).

The second point II $(\bar{\eta}=\sqrt{\alpha}+(\varepsilon / 2 \alpha), D=0)$ corresponds to a homogeneous ordered state «aligned with the external field» and it is a stable node (Fig. 1).

The third point III $(\bar{\eta}=-\sqrt{\alpha}+(\varepsilon / 2 \alpha), D=0)$ corresponds to homogeneous ordering, but it is «opposite to the field». This point is also a stable node (Fig. 1).

Both the second and the third singular points correspond to single-domain types of the specimen ordering.

All the rest of the singular points (with $D \neq 0$ ) correspond to inhomogeneous ordering, i.e., to one or the another polydomain structures.

The fourth point IV $\left(\bar{\eta}=\varepsilon / 2 \alpha ; D=\alpha-3 \varepsilon^{2} / 4 \alpha^{2}\right)$ (Fig. 1) is a stable node and corresponds to the possibility of the polydomain structure realization. The fact that $\bar{\eta} \neq 0$ in this state corresponds to some non-equivalency of domains of two types. However, in weak fields this distinction is small (to the extent of the ratio $\varepsilon / \alpha$ ).

And finally, there are another two singular points of the saddle type. One of them $\mathrm{V}$ («right saddle») with coordinates $(\bar{\eta}=\sqrt{\alpha} / 2-\varepsilon / 4 \alpha ; D=\alpha / 4+3 \varepsilon / 4 \sqrt{\alpha})$ (Fig. 1) corresponds to a possibility for realizing such a quasi-stationary polydomain structure, where the volume fraction of domains of the same type (e.g., with polarization vector, aligned with field) substantially exceeds the volume fraction of the other type domains (opposed to the field).

The other point («left saddle») with coordinates $(\bar{\eta}=-\sqrt{\alpha} / 2-\varepsilon / 4 \alpha ; D=\alpha / 4-3 \varepsilon / 4 \sqrt{\alpha})$ (the point VI, Fig. 1) corresponds to a possibility for realizing the quasi-stationary polydomain structure as well. However, in this situation the volume fraction of domains with the polarization vector oriented opposite to the field substantially exceeds a volume fraction of domains aligned with the field.

Two separatrices, leaving the origin of coordinates and passing through the «left» and «right» saddle points, divide the phase pattern into the three sectors. The upper central sector (1) is the «attraction region» of the inhomogeneous (polydomain) state, the lower right-hand (2) and lower 
left-hand sectors (3) correspond to two attraction regions of homogeneous single-domain states. Depending on the initial conditions $\left(\bar{\eta}_{0}, D_{0}\right)$ the phase trajectories of the system will be located in one of the above-mentioned sectors. This is illustrated on the phase pattern (Fig. 1), where, apart from the singular points founded analytically and the separatrices of the asymptotic system of equations (14), a number of results of numerical integrating (curves $a-c$ ) of the complete system of equations (13) are presented.

If in the initial state the average value of order parameter $\left|\bar{\eta}_{0} \neq 0\right|$ and it is greater than the fluctuations of order parameter, the system will transfer immediately into one of the single-domain states. The sign of the order parameter in the state of thermodynamic equilibrium is determined by what side of the first singular point the value $\bar{\eta}_{0}$ is located on. We emphasize that the availability of external field $(\varepsilon \neq 0)$, with weak inhomogeneity of order parameter, will make the system to go over into single-domain state, even though $\eta_{0}=0$. The choice between two single-domain states is predetermined by the field direction.

The deflection of the system to one or another side in magnitude of the order parameter with respect to the first singular point can occur, in general, for various reasons, both random and determinate character.

If, however, at the initial state inhomogeneities are sufficiently developed and the average value of the order parameter at the initial moment of time $\bar{\eta}_{0}$ is close to $-(\varepsilon / \alpha)$, the developed polydomain structure will be formed in the system over a time $t \sim \alpha^{-1}$. The characteristic size of the domains, just as the characteristic size of the transition layer (domain boundary) between them, will achieve the value of order $\alpha^{-1 / 2}$ by this moment.

Further, in accordance with (12), the domain sizes will grow in keeping with the diffusion law $\sim \sqrt{t}$, while the thickness of the domain boundaries remains unchanged at the level $\alpha^{-1 / 2}$.

Strictly speaking, if the long-range interaction is not counted, the polydomain state is not stable thermodynamically. The state of interest may be considered to be long-lived and its characteristic lifetime is $\alpha^{-1}<<t<d^{2}$. That is, in the situation being considered the system will pass to the thermodynamically stable monodomain state as well. However, this transition does not proceed immediately, but it goes through the stage of forming and growing the domains. This growth proceeds for as long as the domain sizes will become of the order of the crystallite size, when by the influence of external field $\varepsilon$ the system will give the preference to the domain of certain sign.

\section{Conclusions}

In this paper we have used a statistical approach [1] to investigate the ordering dynamics under the second order phase transitions in the presence of the external field. This has allowed us to show that the imposition of a not-too-strong field to the ordering system leads to the asymmetry of the ordering process, removing the degeneracy on the sign of the order parameter, i.e. it makes monodomain states with the order parameters $+\eta$ and $-\eta$ nonequivalent. However both in the weak field and at the absence of the field the formation of a polydomain ordered structure is most likely. Despite its thermodynamic instability, the structure of this kind will evolve rather slowly to the thermodynamic equilibrium monodomain type of ordering. The influence of a weak external field on the polydomain structure lies only in the fact that the volume redistribution of an energetically disadvantageous regions (oriented opposite to the field) and advantageous regions (aligned with the field) will occur in favour of the latter. The homogeneous (monodomain) state of ordering is realized just in sufficiently strong fields in excess of the critical value $\varepsilon_{c}$ (which is generally dependent on the temperature), irrespective of the initial conditions. The field $\varepsilon_{c}$ is nothing but a coercive field

The author thanks to Prof. E. P. Feldman for fruitful discussions. This work was supported in part by the State Foundation of Fundamental Investigations of Ukraine through the Grant No $2.4 / 220-97$.

1. E. P. Feldman and L. I. Stefanovich, Zh. Eksp. Teor. Fiz. 96, 1513 (1989) [Sov. Phys. JETP 69, 858 (1989)].

2. E. P. Feldman and L. I. Stefanovich, Pis'ma Zh. Eksp. Teor. Fiz. 63, 933 (1996) [Sov. Phys. JETP Lett. 63, 983 (1996)].

3. J. W. Cahn and A. Novic-Cohen, J. Stat. Phys. 76, 877 (1994).

4. I. M. Lifshits, Zh. Eksp. Teor. Fiz. 42, 1354 (1962) [Sov. Phys. JETP 15, 939 (1962)].

5. L. D. Landau and E. M. Lifshits, Statistical Physics, Nauka, Moscow (1976) [English translation published by Pergamon Press, Oxford].

6. A. A. Andronov, E. A. Leontovich, I. I. Gordon, and A. G. Mayer, Qualitative Theory of Second-Order Dynamic Systems [in Russian], Fizmatgiz, Moscow (1966). 